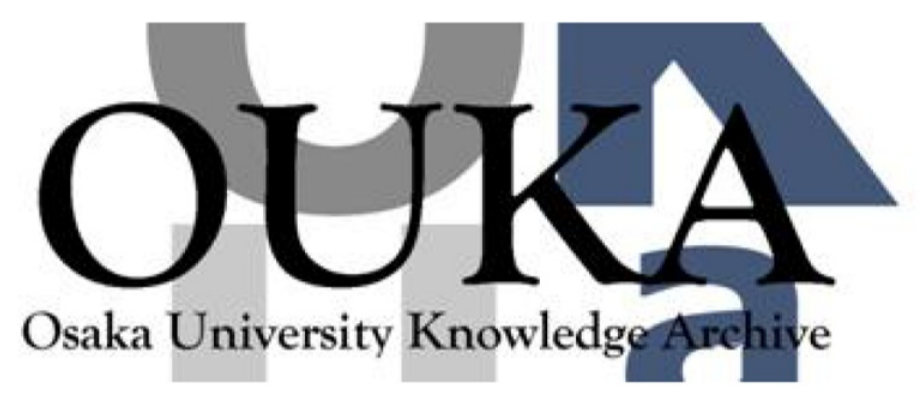

\begin{tabular}{|c|c|}
\hline Title & $\begin{array}{l}\text { Precise control of hydrogen response of } \\
\text { semicontinuous palladium film using } \\
\text { piezoelectric resonance method }\end{array}$ \\
\hline Author (s) & Nakamura, N.; Ueno, T. ; Ogi, H. \\
\hline Citation & $\begin{array}{l}\text { Applied Physics Letters. 114(20) p. 201901-1- } \\
\text { p. 201901-5 }\end{array}$ \\
\hline Issue Date & $2019-05-20$ \\
\hline oaire:version & VoR \\
\hline URL & https://hdl. handle. net/11094/83920 \\
\hline rights & $\begin{array}{l}\text { Copyright } 2019 \text { Authors. This article may be } \\
\text { downloaded for personal use only. Any other use } \\
\text { requires prior permission of the author and AIP } \\
\text { Publishing. This article appeared in Applied } \\
\text { Physics Letters, } 114(20), 201901,2019 \text { and may } \\
\text { be found at https://doi.org/10.1063/1.5094917. }\end{array}$ \\
\hline Note & \\
\hline
\end{tabular}

Osaka University Knowledge Archive : OUKA

https://ir. Library. osaka-u. ac. jp/

Osaka University 


\section{Precise control of hydrogen response of semicontinuous palladium film using piezoelectric resonance method $\odot$}

Cite as: Appl. Phys. Lett. 114, 201901 (2019); https://doi.org/10.1063/1.5094917

Submitted: 06 March 2019 . Accepted: 19 April 2019 . Published Online: 20 May 2019

N. Nakamura, T. Ueno, and H. Ogi

\section{COLLECTIONS}

F This paper was selected as Featured
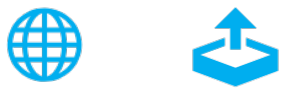

\section{ARTICLES YOU MAY BE INTERESTED IN}

Hydrogen-gas sensing at low concentrations using extremely narrow gap palladium nanoclusters prepared by resistive spectroscopy

Journal of Applied Physics 126, 225104 (2019); https://doi.org/10.1063/1.5119314

Highly sensitive hydrogen detection using curvature change of wireless-electrodeless quartz resonators

Applied Physics Letters 115, 171901 (2019); https://doi.org/10.1063/1.5126135

Fabrication of polymeric lenses using magnetic liquid molds

Applied Physics Letters 114, 203701 (2019); https://doi.org/10.1063/1.5090511

\section{Challenge us.}

What are your needs for periodic signal detection?

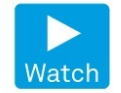

- Zurich

- Instruments

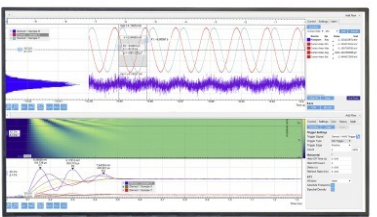

0 


\title{
Precise control of hydrogen response of semicontinuous palladium film using piezoelectric resonance method $\odot$
}

Cite as: Appl. Phys. Lett. 114, 201901 (2019); doi: 10.1063/1.5094917

Submitted: 6 March 2019 • Accepted: 19 April 2019 .

Published Online: 20 May 2019

N. Nakamura, ${ }^{1, a)}$ T. Ueno, ${ }^{1}$ and H. Ogi ${ }^{2}$

\author{
AFFILIATIONS \\ ${ }^{7}$ Graduate School of Engineering Science, Osaka University, 1-3 Machikaneyama, Toyonaka, Osaka 560-8531, Japan \\ ${ }^{2}$ Graduate School of Engineering, Osaka University, 2-1 Yamadaoka, Suita, Osaka 565-0871, Japan

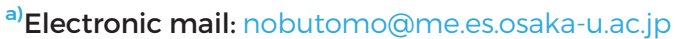

\begin{abstract}
During deposition of metallic material on a substrate, a semicontinuous film composed of isolated and connected clusters is formed transiently at a few nanometers thickness. The surface electrical conductivity at this moment is governed by the tunneling conduction, and slight subsequent evolution of the film morphology changes the conductivity markedly because of the island connection. When the semicontinuous palladium film is exposed to hydrogen, its morphology changes because of absorption of hydrogen, changing the surface conductivity drastically. This phenomenon is applicable for hydrogen sensing. However, it has been significantly difficult to fabricate an optimum semicontinuous structure because it appears in a very short time during deposition. In the present study, we precisely control the palladium film morphology using the piezoelectric resonance method. In this method, an electric field is excited around the substrate surface using the resonant vibration of the piezoelectric material. The electric field generates the electrical current in the deposited material, and the vibrational energy of the piezoelectric material is spent on it. Because the energy loss depends on the electrical conductivity (morphology) of the deposited material, by measuring the change in the attenuation of the resonant vibration, evolution of the morphology is detectable. Using this method, palladium films with several morphologies were fabricated, and the conductivity change in hydrogen was evaluated. The change ratio of the conductivity significantly depended on the morphology, and the conductive sensitivity to the hydrogen detection with the optimum structure was larger than that reported in the previous study by a factor of 12 .
\end{abstract}

Published under license by AIP Publishing. https://doi.org/10.1063/1.5094917

When metallic material is deposited on a substrate, the morphology of the deposited material changes in a very short time: formation and growth of clusters, contact of clusters, and formation of a continuous film. During the growth process, the surface conduction is caused first by tunneling conduction (hopping conduction) between separated clusters and then by the bulk conduction along the connected cluster network, during which the electrical resistance drastically decreases. ${ }^{1,2}$ Around the transition between the dispersed cluster (island) structure (discontinuous film) and the connected cluster structure (continuous film), the semicontinuous film structure composed of separated and connected clusters is transiently formed. In such a film, the surface conductivity is determined by the balance between the tunneling and bulk conduction, and when the distance between clusters is changed because of the cluster growth, the electrical resistance changes markedly.
The morphology-sensitive electrical conductivity of the semicontinuous film is applicable to hydrogen sensing. ${ }^{3-5}$ When the palladium semicontinuous film is exposed to hydrogen, palladium absorbs hydrogen and a hydrogen-palladium alloy is formed. This causes volume expansion, and the gap between separated clusters closes, which decreases the electrical resistance. ${ }^{4,5}$ Closing and opening of nanogaps in nanostructures such as palladium mesowire arrays, ${ }^{6}$ palladium nanoparticle films, ${ }^{7}$ and nanogap palladium films ${ }^{8}$ are also applied for hydrogen sensing. In the nanostructures, the change ratio of the resistance is larger than that observed in the semicontinuous film. However, somewhat large volume change is required to close the gap in the structures. Therefore, they have been applied for detection of hydrogen at high concentrations $(>0.4 \%)$. In contrast, the gap distance between separated clusters becomes less than $1 \mathrm{~nm}$ in the semicontinuous film. Therefore, if we can optimize the gap distance precisely, the hydrogen detection ability (detection limit) is improved. 
However, it has been significantly difficult to fabricate the optimum semicontinuous structure, because it appears in a very short time during deposition.

In previous studies on the semicontinuous palladium film, the morphology was characterized by measuring the temperature coefficient of the resistance ${ }^{5}$ and by observing the morphology using atomic force microscopy ${ }^{4}$ after the film was deposited. The in situ conductivity measurement during deposition was also utilized. ${ }^{4}$ During deposition, formation of the continuous film is completed below $\sim 4 \mathrm{~nm}$, and the discontinuous-continuous transition occurs within 1-nm thickness change as observed in the following experiment. To interrupt the deposition precisely at the moment where the desired morphology is formed, in situ structural characterization methods are needed. The resistance measurement, curvature measurement, ${ }^{9-11}$ and differential reflectance spectroscopy ${ }^{12}$ are applicable for the in situ monitoring. In contrast, in the present study, we use the piezoelectric resonance method. ${ }^{13}$ This method was developed in our previous work, and it clearly detects the moment of the discontinuous-continuous transition during deposition. This feature is suitable for optimizing the semicontinuous structure. Using this method, we fabricate palladium films with a variety of morphologies and evaluate the relationship between the morphology and hydrogen response. It is then demonstrated that a slight difference in the morphology changes the hydrogen response significantly, and the piezoelectric resonance method allows us to obtain palladium films with a desired morphology and hydrogen response.

Palladium (99.95\%) was deposited on the silicon (001) substrate ( $>10000 \Omega \mathrm{cm}$ ) by the RF magnetron sputtering. The thickness of the substrate was $100 \mu \mathrm{m}$, and the in-plane dimension was $7 \mathrm{~mm} \times 5 \mathrm{~mm}$. The substrate surface was naturally oxidized. The background pressure was less than $2.0 \times 10^{-4} \mathrm{~Pa}$, and the argon pressure during deposition was $0.4 \mathrm{~Pa}$. The sputtering power was $20 \mathrm{~W}$.

As shown in Fig. 1(a), the silicon substrate was placed on the sample holder, and palladium was deposited on the top surface of the substrate. In the sample holder, a piezoelectric material and antennas are embedded. The piezoelectric material used here is rectangular parallelepiped lithium niobate, measuring $2.5 \mathrm{~mm} \times 1.7 \mathrm{~mm} \times 0.2 \mathrm{~mm}$ along the $X, Y$, and $Z$ directions, respectively. During deposition, the lithium niobate was oscillated with changing the frequency, and the resonant spectrum of its free vibration was measured repeatedly using the antennas. ${ }^{14,15}$ The vibrating lithium niobate generates the electric field near the substrate surface, and it causes the electrical current flow in the deposited palladium. When separated clusters are formed on the substrate, the surface resistance is significantly high, and energy loss by Joule heating barely occurs. As the cluster grows and the gap distance becomes smaller, the resistance decreases and the current is increased, in which the energy loss becomes larger. After clusters connect each other and a continuous film is formed, the resistance becomes significantly small, and the energy loss becomes smaller. Therefore, the full width at half maximum (FWHM) of the resonant peak shows maximum at the discontinuous-continuous transition, which precisely tells us the moment of the transition. The details of the measurement principle and setup are described elsewhere. ${ }^{13,16}$

The resonant spectrum was measured around $60 \mathrm{MHz}$ using the network analyzer, and a representative spectrum is shown in Fig. 1(b). The frequency is much higher than the resonant frequency $(\sim 0.18 \mathrm{MHz})$ of the fundamental mode of the

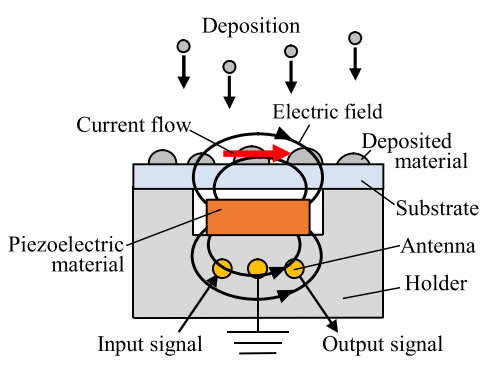

(a)

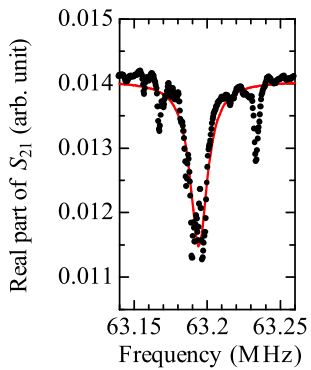

(b)

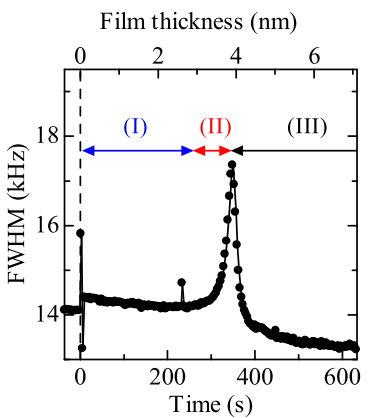

(c)

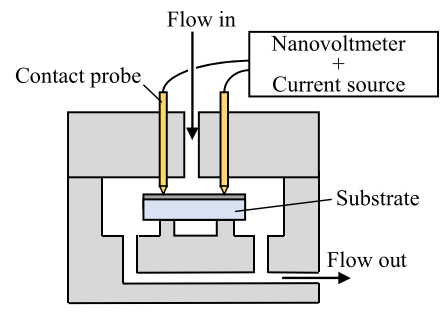

(d)
FIG. 1. (a) Schematic image of the simplified measurement setup. (b) Resonant spectra measured by the network analyzer. Plots denote the measured data and the line denotes the fitted Lorentz function. (c) Evolution of FWHM during deposition of palladium on a silicon substrate. (d) Schematic image of the hydrogen flow cell.

rectangular-parallelepiped lithium niobate; the frequency was calculated using the Ritz method ${ }^{17}$ using the reported elastic and piezoelectric properties. ${ }^{18}$ Because silicon is a semiconductor, current flows in the silicon substrate when the film resistance is high. This indicates that the change in the film morphological cannot be detected. This problem is solved by measuring higher resonant modes. This is because electrical impedance of the silicon substrate is increased when the frequency of the alternating current generated by the vibrating lithium niobate is increased. ${ }^{13}$ The current, then, flows in deposited palladium at high frequencies, and the FWHM peak is successfully observed as shown in Fig. 1(c). FWHM was determined by fitting the Lorentz function to the resonant spectrum as shown in Fig. 1(b).

After the deposition, the silicon substrate was set into the hydrogen flow cell [Fig. 1(d)]. Two contact probes were attached to the substrate surface, and the surface resistance was measured with the two-terminal sensing. The distance between the probes was $6 \mathrm{~mm}$. During the hydrogen exposure experiment, nitrogen was flowed as a carrier gas in the cell. Its flow rate was $117 \mathrm{ml} / \mathrm{min}$. Hydrogen detection was performed by adding hydrogen into the carrier gas. The hydrogen gas is composed of $0.1 \%$ hydrogen and $99.9 \%$ nitrogen, and its flow rate was $13 \mathrm{ml} / \mathrm{min}$. Therefore, during the hydrogen flow, the hydrogen concentration was $100 \mathrm{ppm}$ and the flow rate was $130 \mathrm{ml} / \mathrm{min}$ in the cell. The duration time of the hydrogen flow was 408 s. Experiments were performed at room temperature.

We here classify the film-growth process into three stages as shown in Fig. 1(c); (I) before FWHM starts to increase, (II) during 
FWHM increases, and (III) after FWHM shows a maximum. Several palladium films were fabricated by interrupting deposition at different times in each stage. Representative evolutions of FWHM in these stages are plotted in Figs. 2(a), 2(c), and 2(e). Figures 2(b), 2(d), and 2(f) show the resistance changes during hydrogen exposures. $R_{0}$ is the resistance before the film was exposed to hydrogen. In palladium films in stage (I), just after the hydrogen flow starts, the resistance increased rapidly [Fig. 2(b)]. Then, it decreased gradually with time. The change ratio became smaller as $R_{0}$ decreased. In palladium films in stage (II), after a slight increase in the resistance, the resistance decreased sharply with time [Fig. 2(d)]. In some films, the resistance increased again, but the value after $408 \mathrm{~s}$ exposure tended to be smaller than the initial resistance $R_{0}$. In palladium films in stage (III), the resistance decreased monotonically, and the change ratio became smaller as $R_{0}$ decreased [Fig. 2(f)]. A palladium film with $34 \Omega$ was prepared by depositing palladium for $1800 \mathrm{~s}$, and it is supposed to be a completely continuous film.
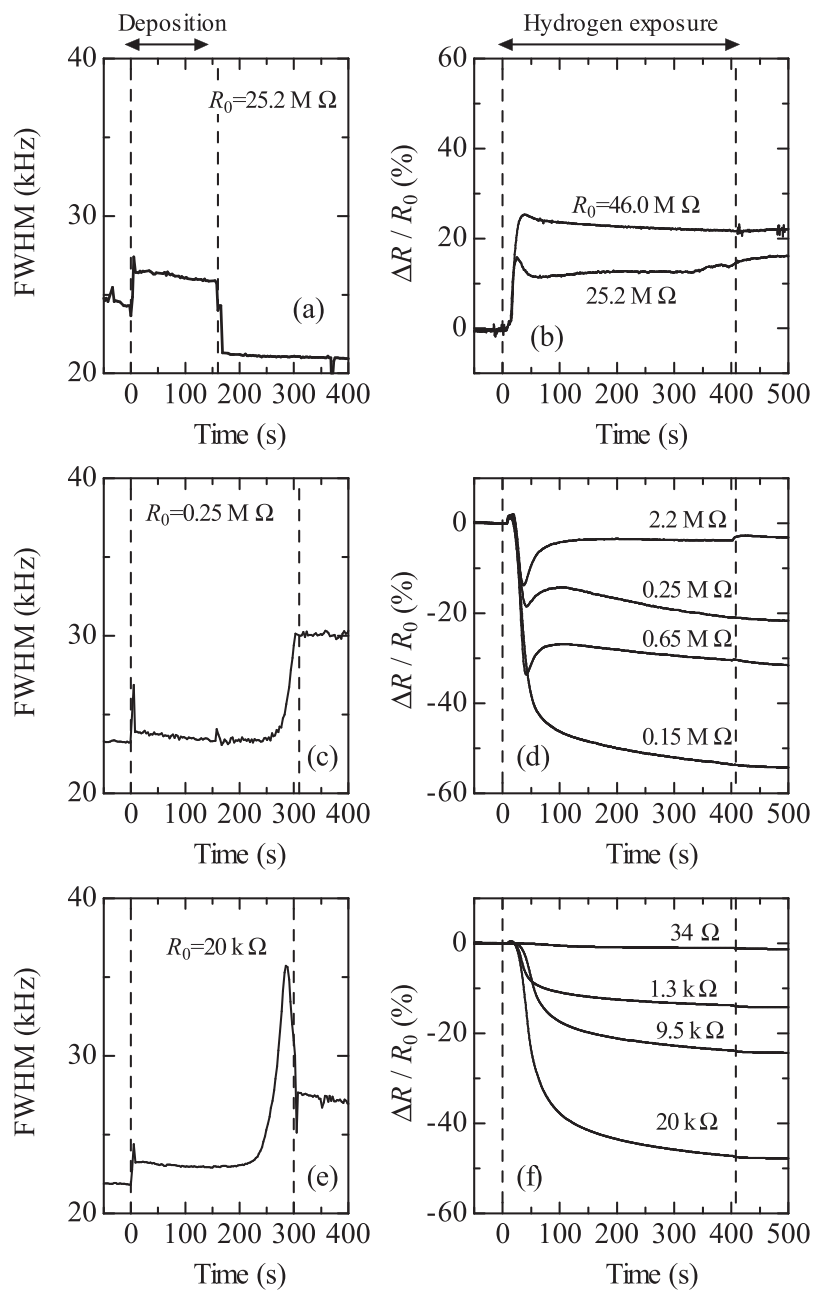

FIG. 2. Changes in FWHM during deposition and hydrogen response. (a) and (b) show the results for palladium films in stage (I), (c) and (d) show the results for palladium films in stage (II), and (e) and (f) show the results for palladium films in stage (III).
The change ratio of the resistance after 408 s exposure is summarized in Fig. 3. It shows that the resistance increases in stage (I), whereas it decreases in stages (II) and (III). In addition, the change ratio becomes maximum at the transition between stages (II) and (III). The maximum change ratio was $53.7 \%$. This is larger than the change ratio, $\sim 4.5 \%$, measured in the previous study using the same type of palladium film at $100 \mathrm{ppm}$.

When palladium is exposed to hydrogen, hydrogen is adsorbed on the palladium surface and it is then absorbed in the palladium. ${ }^{19}$ This reaction process indicates that the resistance change by adsorption appears first, and the change by absorption appears later. Considering this process, an observed hydrogen response is discussed below. In stage (I), separated clusters are formed on the substrate, in which the electrical conductivity is governed by the tunneling (hopping) conductivity as shown in Fig. 4(a). Because the work function of palladium is increased by hydrogen adsorption, ${ }^{19}$ the resistance increases just after the hydrogen exposure starts. Then, the hydrogen absorption occurs, and it increases the volume of the cluster. Then, the gap distance between separated clusters becomes smaller, which decreases the resistance. In the films in stage (II), the gap distance is very small, and some clusters come in contact with each other [Fig. 4(b)]. In the semicontinuous film, the resistance increases quickly by hydrogen adsorption as observed in stage (I). However, expansion of palladium clusters closes the gap, and it decreases the resistance abruptly. As the FWHM approaches the peak in Fig. 1(c), a larger number of gaps are closed by hydrogen absorption, and the change ratio becomes larger. After the FWHM shows a peak [stage (III)], the fraction of the separated clusters becomes small, and the bulk conduction becomes dominant [Fig. 4(c)]. As the film thickness increases, the fraction of separated clusters decreases and a decrease in the resistance also becomes smaller. Thus, the hydrogen response of the palladium film is explained by the balance between the tunneling conductivity and bulk conductivity. It was also demonstrated that the hydrogen response of the palladium film can be controlled using the piezoelectric resonance method.

For representative specimens, hydrogen exposure was repeated a couple of times. Figure 5 compares the hydrogen responses in the first and following exposures. As seen in Figs. 2(b), 2(d), and 2(f), the resistance was almost stable after hydrogen exposures were finished, which

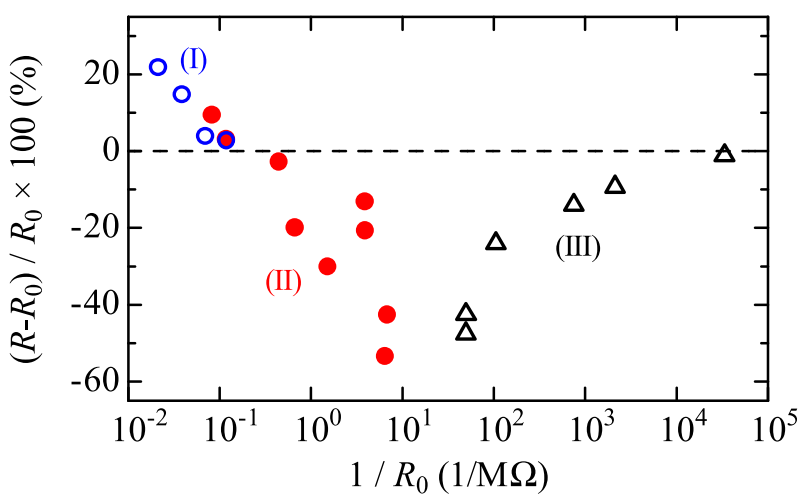

FIG. 3. Change ratio of resistance after hydrogen exposure at $100 \mathrm{ppm}$ for $408 \mathrm{~s}$. Open circles, filled circles, and open triangles denote results for palladium films in stages (I), (II), and (III), respectively. 
(a)

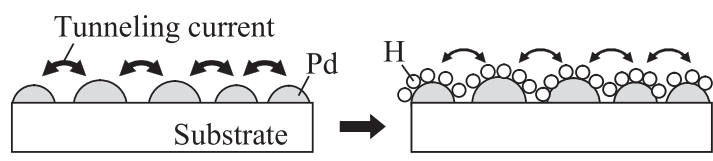

Hydrogen (first)

Bulk conduction

(b)

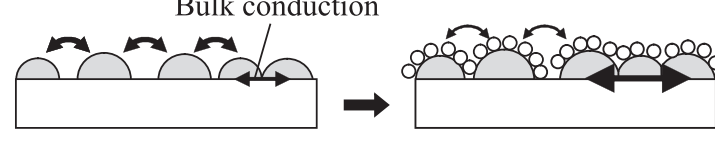

$\Rightarrow$
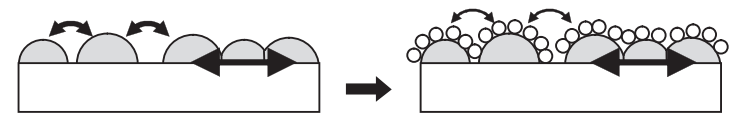

$\Rightarrow$
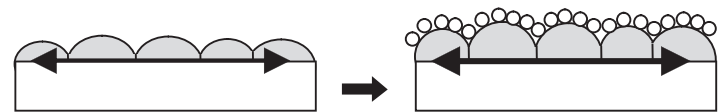

(c)
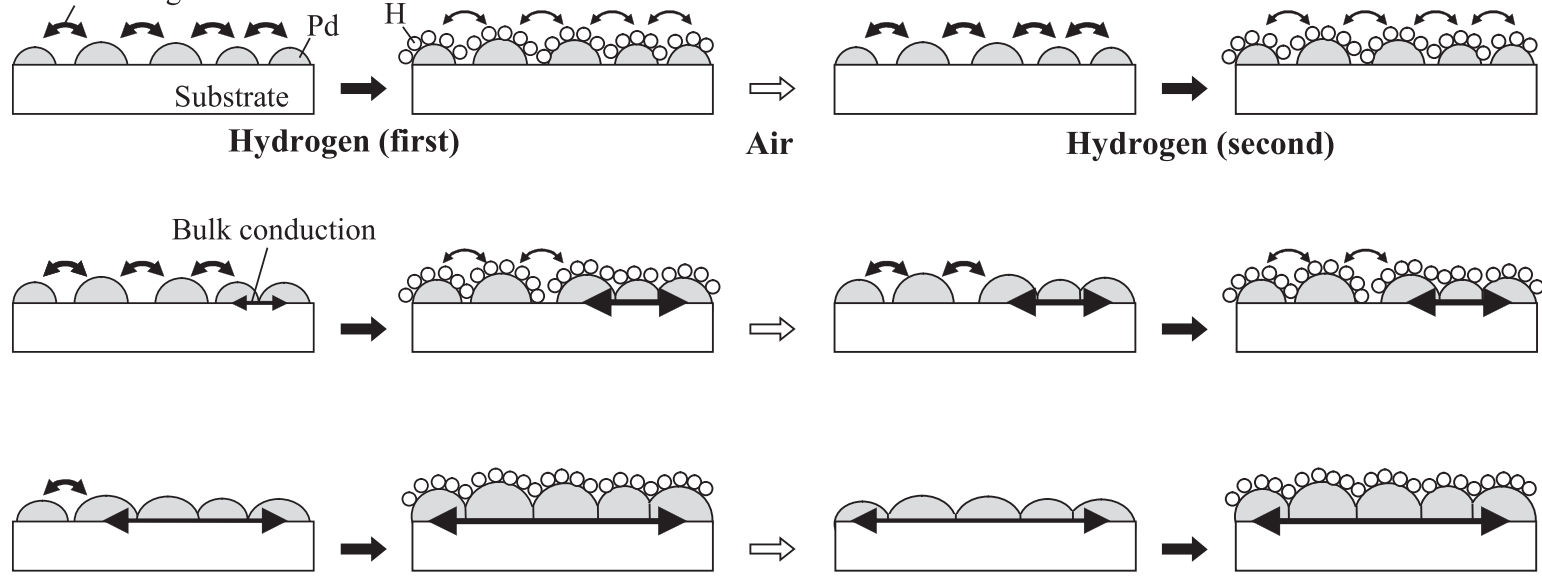

Hydrogen (second)

Air

FIG. 4. Schematic image of the morphological change under repetitive hydrogen exposures for palladium films in (a) stage (I), (b) stage (II), and (c) stage (III). indicates that hydrogen was hardly removed from palladium in nitrogen. On the palladium surface covered with oxygen, oxygen can be removed by water formation with hydrogen. ${ }^{20}$ To remove hydrogen inversely, air in the laboratory was added into the carrier gas, and it was flowed in the cell between each hydrogen exposure.

In stage (I) [Fig. 5(a)], the resistance increased in the following hydrogen exposures as observed in the first hydrogen exposure. This indicates that the morphology of the discontinuous film is hardly changed by the hydrogen exposure, and adsorption of hydrogen dominantly affects the conductivity. It was also confirmed that hydrogen on the palladium surface was removed by the air flow. In contrast, the hydrogen response in stage (II) showed different behaviors between the first and following exposures [Fig. 5(b)]. The resistance increased in the second and following exposures, whereas the resistance decreased in the first exposure. In this stage, the fraction of separated clusters is larger than that of contacting clusters [Fig. 4(b)]. However, during the first hydrogen exposure, some gaps are closed. Once the gaps are closed, they do not open again, and separated clusters with large gaps remain. As observed in the $34 \Omega$ film in Fig. 2(f), the resistance of the continuous film is insensitive to the hydrogen. Therefore, in the second and following hydrogen exposures, the resistance change is governed by the tunneling conduction. In stage (III) [Fig. 5(c)], the resistance decreased in the second and following exposures, as
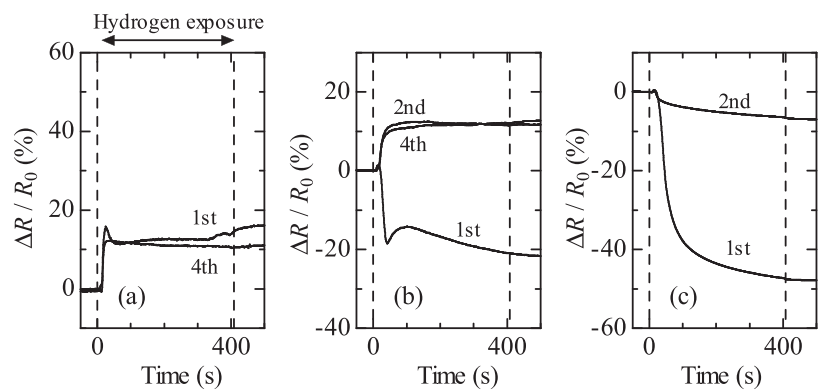

FIG. 5. Comparison of hydrogen response of palladium films in (a) stage (I), (b) stage (II), and (c) stage (III). observed in the first exposure. However, the change ratio decreased. In the films, most of the clusters are connected to each other, and there are a small number of separated clusters. In the first hydrogen exposure, the gaps are closed. In the following exposures, the expansion of palladium clusters by hydrogen absorption increases the contacting area at boundaries (grain boundaries), and the resistance decreases. Formation of a hydrogen-palladium alloy by absorption of hydrogen increases the electrical resistance. ${ }^{21,22}$ However, it was not observed in the films used in the present study.

The palladium films in Figs. 5(b) and 5(c) were prepared by interrupting deposition during the FWHM increases and decreases as shown in Figs. 2(c) and 2(e), respectively. The difference in their thickness is supposed to be only about $0.5 \mathrm{~nm}$, but their hydrogen response was quite different, especially in the second exposure. These results indicate that precise control of deposition is important for obtaining a desired hydrogen response, and it is demonstrated that the piezoelectric resonance method can achieve it.

This research was partially supported by JSPS KAKENHI Grant No. 18H01883. A part of this study was also supported by the Development of Advanced Measurement and Analysis Systems from Japan Science and Technology Agency, JST.

\section{REFERENCES}

${ }^{1}$ I. M. Rycroft and B. L. Evans, Thin Solid Films 290-291, 283-288 (1996).

${ }^{2}$ E. Byon, T. W. H. Oates, and A. Anders, Appl. Phys. Lett. 82, 1634-1636 (2003).

${ }^{3}$ O. Dankert and A. Pundt, Appl. Phys. Lett. 81, 1618-1620 (2002).

${ }^{4}$ T. Xu, M. P. Zach, Z. L. Xiao, D. Rosenmann, U. Welp, W. K. Kwok, and G. W. Crabtree, Appl. Phys. Lett. 86, 203104 (2005).

${ }^{5}$ T. Kiefer, L. G. Villanueva, F. Fargier, F. Favier, and J. Brugger, Appl. Phys. Lett. 97, 121911 (2010).

${ }^{6}$ F. Favier, E. C. Walter, M. P. Zach, T. Benter, and R. M. Penner, Science 293, 2227-2231 (2001).

${ }^{7}$ B. Xie, L. Liu, X. Peng, Y. Zhang, Q. Xu, M. Zheng, T. Takiya, and M. Han, J. Phys. Chem. C 115, 16161-16166 (2011).

${ }^{8}$ M. Zhao, M. H. Wong, and C. W. Ong, Appl. Phys. Lett. 107, 033108 (2015).

${ }^{9}$ R. Abermann, R. Kramer, and J. Mäser, Thin Solid Films 85, 215-229 (1978). 
${ }^{10}$ A. L. Shull and F. Spaepen, J. Appl. Phys. 80, 6243-6256 (1996).

${ }^{11}$ J. A. Floro, S. J. Hearne, J. A. Hunter, P. Kotula, E. Chason, S. C. Seel, and C. V. Thompson, J. Appl. Phys. 89, 4886-4897 (2001).

${ }^{12}$ G. Abadias, L. Simonot, J. J. Colin, A. Michel, S. Camelio, and D. Babonneau, Appl. Phys. Lett. 107, 183105 (2015).

${ }^{13}$ N. Nakamura and H. Ogi, Appl. Phys. Lett. 111, 101902 (2017).

${ }^{14} \mathrm{H}$. Ogi, K. Motohisa, T. Matsumoto, K. Hatanaka, and M. Hirao, Anal. Chem. 78, 6903-6909 (2006).

${ }^{15} \mathrm{~N}$. Nakamura, M. Sakamoto, H. Ogi, and M. Hirao, Rev. Sci. Instrum. 83, 073901 (2012)
${ }^{16}$ N. Nakamura, N. Yoshimura, H. Ogi, and M. Hirao, J. Appl. Phys. 118, 085302 (2015).

${ }^{17}$ I. Ohno, Phys. Chem. Miner. 17, 371-378 (1990).

${ }^{18}$ H. Ogi, Y. Kawasaki, M. Hirao, and H. Ledbetter, J. Appl. Phys. 92, 2451-2456 (2002).

${ }^{19} \mathrm{H}$. Conrad, G. Ertl, and E. E. Latta, Surf. Sci. 41, 435-446 (1974).

${ }^{20}$ D. Gupta, D. Dutta, M. Kumar, P. B. Barman, C. K. Sarkar, S. Basu, and S. K. Hazra, Sens. Actuators B 196, 215-222 (2014).

${ }^{21}$ W. T. Lindsay and F. W. Pement, J. Chem. Phys. 36, 1129-1234 (1962).

${ }^{22}$ R. J. Smith and D. A. Otterson, J. Phys. Chem. Solids 31, 187-189 (1970). 